\title{
Traumatic Posterior Fossa Hematoma, A Rare Entity: Study of 21 Cases
}

\author{
Sandeep Bhardwaj ${ }^{1}$ Vinod Sharma ${ }^{1} \quad$ Somnath Sharma ${ }^{1} \quad$ Devendra Purohit ${ }^{1} \quad$ Sanjeev Chopra ${ }^{1}$ \\ ${ }^{1}$ Department of Neurosurgery, SMS Hospital, Jaipur, Rajasthan, \\ India \\ Address for correspondence Vinod Sharma, MBBS, MS, MCh, Room \\ No. F 13, Resident Doctors Hostel, SMS Hospital, Jaipur, Rajasthan, \\ India (e-mail: neurovinod@gmail.com).
}

J Neurosci Rural Pract 2019;10:675-682

\begin{abstract}
Keywords

- traumatic posterior fossa hematoma

- subarachnoid hemorrhage

- Glasgow Coma Scale

Background Traumatic posterior fossa hematoma is a rare entity. Traumatic posterior fossa hematomas are associated with considerable morbidity and mortality and their surgical management remained controversial.

Methods From August 2011 to August 2017, approximately 5,100 patients with head injury were managed. Authors reviewed clinical and radiological findings, management criteria, and outcome of posterior fossa hematoma in 21 patients.

Results Out of 21 cases, 13 survived with our management. The Glasgow Coma Scale (GCS) on admission was higher in favorable group than in poor outcome group. Factors associated with Glasgow Outcome Scale in two groups were status of fourth ventricle, basal cisterns, subarachnoid hemorrhage (SAH), hematoma volume, and their location (hemispheric or midline). Similarly, associated supratentorial lesions, age, gender, lesions in other parts of body, and timing from injury to reporting to hospital were taken into consideration.

Conclusion The factors correlated with patient outcome were age, sex, mode of injury, GCS at admission, associated intracranial hematomas, associated SAH, hematoma volume, hematoma location, basal cisterns, status of fourth ventricle, and associated multiple injuries on other body parts. It is hereby concluded that timely surgical intervention should be employed whenever indicated without delay. Posterior fossa hematomas were rarely observed in the pediatric age group.
\end{abstract}

\section{Introduction}

Traumatic posterior fossa hematoma is very less as compared with spontaneous hematomas associated with hypertension. They are associated with significant mortality and morbidity. They constitute a small subset of total head injury patients. ${ }^{1}$ As we know, posterior fossa have small volume, and any increase in volume due to any cause may cause increase intracranial pressure, herniation, and deterioration. Traumatic posterior fossa hematomas account for $<1 \%$ in all head injury patients. ${ }^{2,3} \mathrm{Liu}^{4}$ reported the incidence of traumatic posterior fossa hematoma to be $3.7 \%$ of total intracranial hematomas. These hematomas may be totally asymptomatic, with a sudden increase in size can lead to rapid deterioration of neurological status. ${ }^{5}$
Traumatic cerebellar hematoma may be isolated or may be associated with subdural hemorrhage (SDH), extradural hemorrhage (EDH), and subarachnoid hemorrhage (SAH) in posterior fossa. They may present acutely or in delayed manner. The hematoma location, volume, initial Glasgow Coma Scale (GCS) score, status of fourth ventricle, and basal cisterns are important in final outcome. Management of these hematomas nowadays have changed from all surgery to conservative management. ${ }^{1}$

Authors evaluated outcome of patients on GCS score at admission, location, volume, status of cisterns, fourth ventricle, $\mathrm{SAH}$, and supratentorial lesions. The surgical indications were posterior fossa hematoma with mass effect, midline shift, size greater than $3 \mathrm{~cm}$, effaced basal cisterns, fourth ventricle compression, and associated supratentorial lesions with significant mass effect with midline shift. 
Similarly, patients were managed conservatively with respect to hematoma location, size less than $3 \mathrm{~cm}$, normal basal cisterns, and fourth ventricle. In some of these hematomas, early surgical intervention is deemed necessary..$^{5-9}$ The early results of surgery are good, so surgical intervention if required should not be delayed. The course and prognosis of midline hematoma is worse, so evacuation should be done early.

\section{Methods}

From August 2011 to August 2017, approximately 5,100 patients with head injury were admitted in our institute. This study was focused on 21 patients (0.41\%) with traumatic posterior fossa hematomas. The clinical findings, mode of injury, initial GCS, various radiological parameters, and final outcome were evaluated in 21 patients using Glasgow Outcome Scale (GOS). GOS was graded into good recovery (GR), moderate disability, severe disability (SD), vegetative state (VS), and death (D).

Initial computed tomography (CT) head was obtained at admission of patient and repeat CT head was done at 6 hours if the patient deteriorated more than 2 points of initial GCS score at admission. On CT scan, hematoma location, size, status of basal cisterns (normal or compressed), status of fourth ventricle (normal or compressed), associated $\mathrm{SAH}$, and supratentorial lesions were evaluated. Hematoma volume was assessed by using formula $A^{*} B^{*} C / 2$, where $A$ is maximum transverse diameter of hemorrhage on CT, B is anterioposterior diameter, and $\mathrm{C}$ is number of CT slices showing hematoma. ${ }^{10}$

Hematomas were classified as type 1 vermian hematoma (-Fig. 1) and type 2 hemispheric hematoma involving superficial two-thirds of the hemisphere (-Figs. 2 and 3). Injury is classified as coup, countercoup, and acceleration-deceleration type. Time to reach hospital is important as it affects overall morbidity and mortality of patient.

\section{Results}

\section{Clinical and Radiological Findings}

Out of 21 patients, 18 were male and the rest females. Most of the patients were in the age range of 30 to 60 years. The mode of injury was road traffic accident (15 patients) and fall ( 6 patients). Duration between sustaining injury and reporting to hospital was 4 hours in 11 patients and 5 to 8 hours in 10 patients. GCS score on admission ranged from 3 to 15 as shown in - Table 1. The location of hematoma was type 1 ( 8 patients) and type 2 (13 patients). Nine patients had isolated posterior fossa hematoma and 12 patients presented with associated intracranial lesions.

Intracranial lesions were supratentorial contusion (11), SDH (2), EDH (2), SAH (9), IVH (1), diffuse axonal injury (DAI) (1), hydrocephalus (HCP) (2), and SDH in posterior fossa in 1 patient ( $\mathbf{- F i g . 3 C}$ ). Basal cisterns were compressed in 9 and

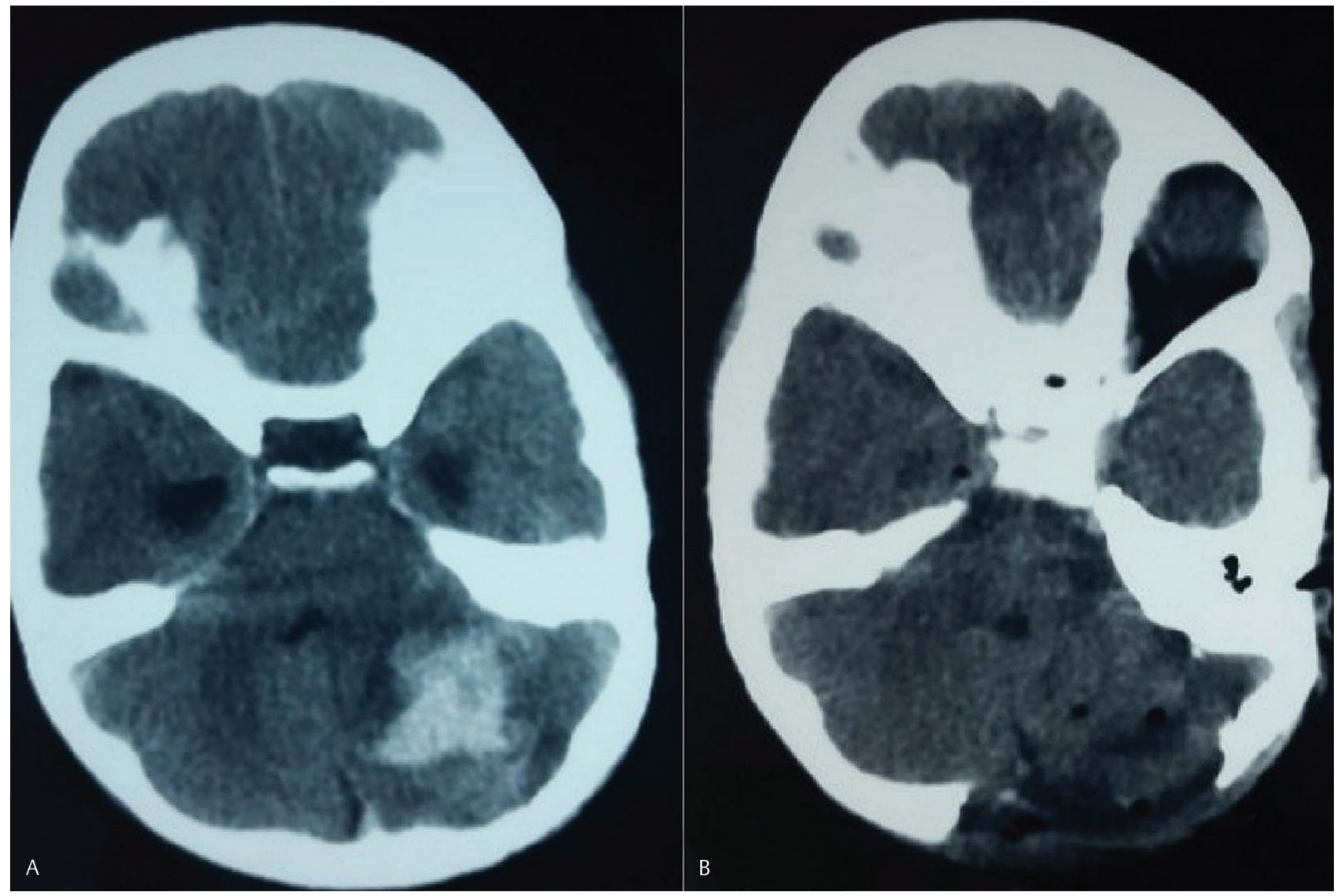

Fig. 1 (A) Computerized tomography scan revealing vermian hematoma (type 1). (B) Postoperative scan after suboccipital craniectomy and hematoma evacuation (type 1 ). 


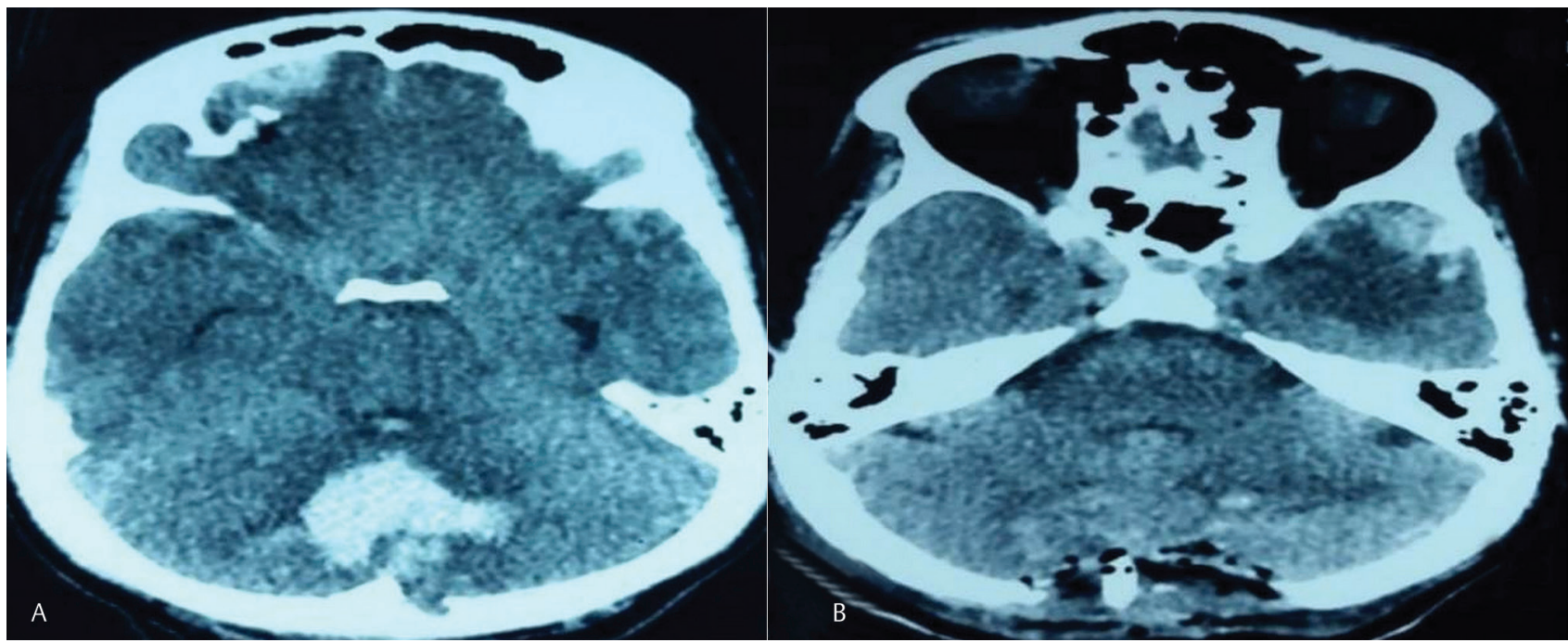

Fig. 2 (A) Computerized tomography scan revealing preoperative image type 2 hematoma. (B) Postoperative scan showing complete removal of hematoma.

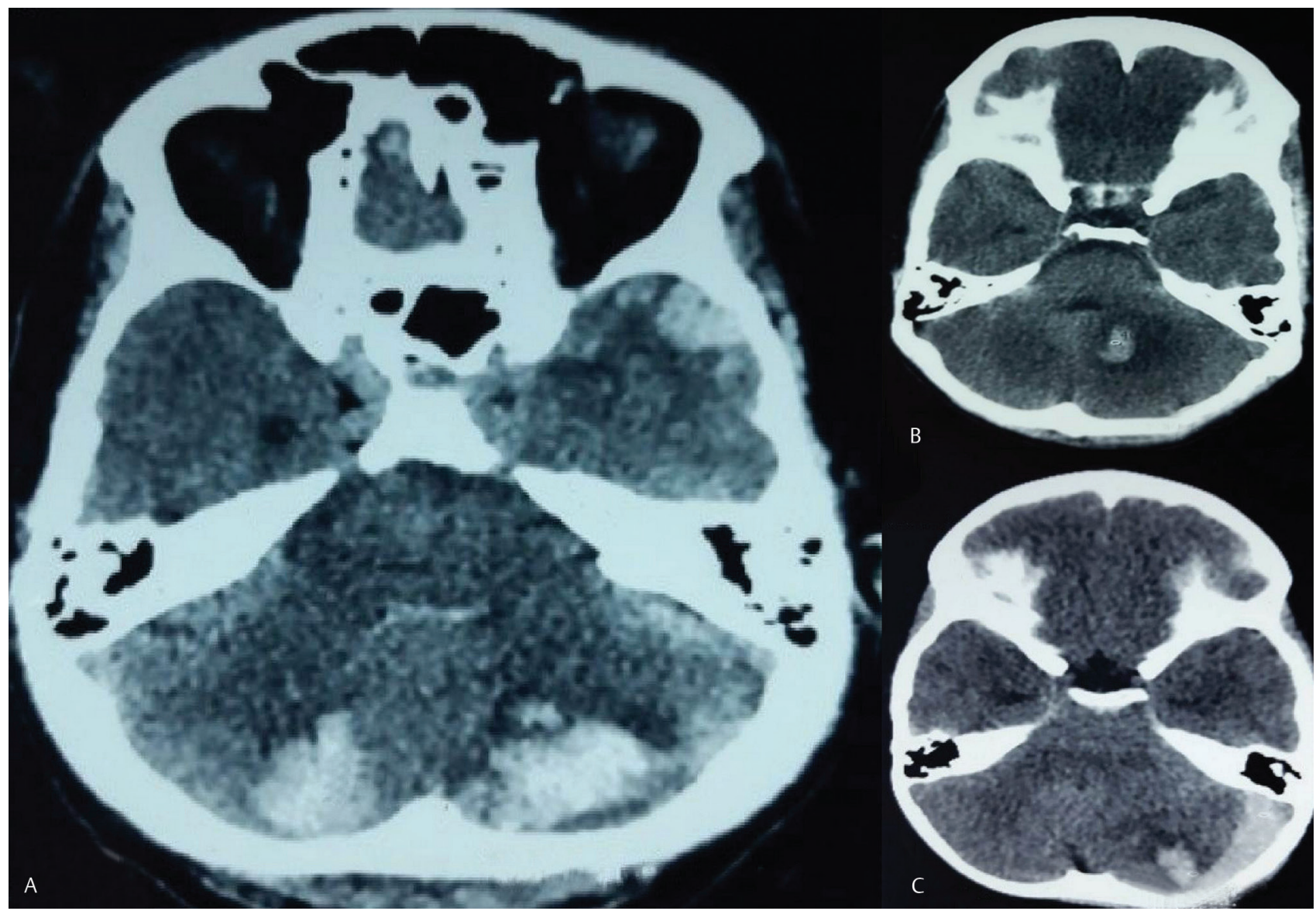

Fig. 3 (A) Computerized tomography scan revealing bilateral type 2 hematoma. (B) Computerized tomography revealing type 2 hematoma. (C) Computerized tomography revealing type 2 hematoma with subdural hemorrhage.

normal in 12 patients. Fourth ventricle had intraventricular hemorrhage (IVH) in 3, was compressed in 3, and normal in 15 patients. Associated injuries were found in 8 , and skull fracture was seen in 15 patients. Out of 8 patients, only 1 had splenic injury which was managed conservatively and 1 patient had hemothorax which was managed by intercostal tube drainage.

\section{Type 1 and Type 2 Hematoma Outcome}

Comparison between type 1 and 2 hematomas is shown in - Table 2. Age range in type 1 was 22 to 56 years, while in type 2 hematomas it was 12 to 79 years. In type 2 hematomas, age varied over a wider range as compared with type 1. Volume of hematoma was higher in type 2 hematomas as 
Table 1 Summary of cases

\begin{tabular}{|c|c|c|c|c|c|c|c|c|c|c|c|c|c|c|}
\hline $\begin{array}{l}\text { S. } \\
\text { no. }\end{array}$ & $\begin{array}{l}\text { Age } \\
(y)\end{array}$ & $\begin{array}{l}\text { Sex } \\
(M / F) \\
\text { Dura- } \\
\text { tion }^{a}\end{array}$ & $\begin{array}{c}\text { Mode of } \\
\text { injury, } \\
\text { skull } \\
\text { fracture }\end{array}$ & $\begin{array}{c}\text { Asso- } \\
\text { ciated } \\
\text { lesions }\end{array}$ & $\begin{array}{c}\text { Site of } \\
\text { impact }\end{array}$ & $\begin{array}{c}\text { GCS } \\
(3-15)\end{array}$ & $\begin{array}{c}\text { Volume, } \\
\mathrm{cm}^{3}, \text { size } \\
(\mathrm{cm})\end{array}$ & $\begin{array}{c}\text { Type of } \\
\text { location } \\
(1 / 2)\end{array}$ & $\begin{array}{c}\text { Basal } \\
\text { cis- } \\
\text { terns } \\
(\mathrm{N} / \mathrm{C})\end{array}$ & $\begin{array}{c}\text { Fourth } \\
\text { ventricle } \\
(\mathrm{N} / \mathrm{C} / \mathrm{IVH})\end{array}$ & $\begin{array}{c}\text { Associated } \\
\text { supratento- } \\
\text { rial lesions }\end{array}$ & $\begin{array}{l}\mathrm{SAH} \\
(\mathrm{A} / \mathrm{P})\end{array}$ & Intervention & GOS \\
\hline 1 & 40 & $\mathrm{M}, 4 \mathrm{~h}$ & MVA,- & None & $\begin{array}{l}\text { Occip- } \\
\text { ital }\end{array}$ & $14 / 15$ & \begin{tabular}{|l|}
16.8 \\
$(3.5 \mathrm{~cm})$
\end{tabular} & 2 & $\mathrm{~N}$ & IVH & No lesion & A & $\begin{array}{l}\text { Suboccipital } \\
\text { craniectomy }\end{array}$ & GR \\
\hline 2 & 40 & $\mathrm{M}, 6 \mathrm{~h}$ & MVA,+ & None & Frontal & $5 / 15$ & $4(2)$ & 1 & C & $N$ & $\begin{array}{l}\text { Right } \\
\text { frontal } \\
\text { contusion }\end{array}$ & $P$ & Conservative & D \\
\hline 3 & 30 & F, $7 \mathrm{~h}$ & MVA,+ & None & $\begin{array}{l}\text { Fron- } \\
\text { tal, } \\
\text { occip- } \\
\text { ital }\end{array}$ & $6 / 15$ & $19.2(4)$ & 2 & $C$ & $N$ & $\begin{array}{l}\text { Right fron- } \\
\text { to-tempo- } \\
\text { ro-parietal } \\
\text { acute SDH } \\
\text { with IVH }\end{array}$ & $P$ & $\begin{array}{l}\text { Subocipital } \\
\text { craniectomy }\end{array}$ & D \\
\hline 4 & 12 & M, $2 \mathrm{~h}$ & MVA,- & None & $\begin{array}{l}\text { Occip- } \\
\text { ital }\end{array}$ & $15 / 15$ & $2(2)$ & 2 & $N$ & $N$ & No lesion & A & Conservative & GR \\
\hline 5 & 65 & $\mathrm{M}, 4 \mathrm{~h}$ & Fall,+ & $\begin{array}{l}\text { Abdom- } \\
\text { inal } \\
\text { injury }\end{array}$ & $\begin{array}{l}\text { Occip- } \\
\text { ital }\end{array}$ & $12 / 15$ & $\begin{array}{l}15.4 \\
(3.5)\end{array}$ & 2 & $\mathrm{~N}$ & $N$ & No lesion & A & $\begin{array}{l}\text { Suboccipital } \\
\text { craniectomy }\end{array}$ & MR \\
\hline 6 & 47 & $\mathrm{M}, 2 \mathrm{~h}$ & MVA,+ & $\begin{array}{l}\text { Frac- } \\
\text { ture left } \\
\text { ulna }\end{array}$ & $\begin{array}{l}\text { Occip- } \\
\text { ital }\end{array}$ & $13 / 15$ & $40(5)$ & 2 & N & C & No lesion & A & $\begin{array}{l}\text { Suboccipital } \\
\text { craniectomy }\end{array}$ & MR \\
\hline 7 & 35 & M, $3 \mathrm{~h}$ & Fall,- & None & $\begin{array}{l}\text { Occip- } \\
\text { ital }\end{array}$ & $13 / 15$ & $\begin{array}{l}15.4 \\
(3.5)\end{array}$ & 2 & $\mathrm{~N}$ & $N$ & No lesion & A & $\begin{array}{l}\text { Suboccipital } \\
\text { craniectomy }\end{array}$ & GR \\
\hline 8 & 75 & $\mathrm{~F}, 6 \mathrm{~h}$ & Fall,+ & None & Occiput & $7 / 15$ & $19.2(4)$ & 2 & $\mathrm{~N}$ & C & No lesion & $P$ & $\begin{array}{l}\text { Suboccipital } \\
\text { craniectomy }\end{array}$ & D \\
\hline 9 & 22 & $\mathrm{M}, 8 \mathrm{~h}$ & MVA,+ & $\begin{array}{l}\text { Frac- } \\
\text { ture } \\
\text { femur }\end{array}$ & $\begin{array}{l}\text { Fron- } \\
\text { tal, } \\
\text { occip- } \\
\text { ital }\end{array}$ & $5 / 15$ & $4(2)$ & 1 & C & $N$ & $\begin{array}{l}\text { Left frontal } \\
\text { EDH, with } \\
\text { bilateral } \\
\text { temporal } \\
\text { contusions, } \\
\text { with hydro- } \\
\text { cephalus }\end{array}$ & $P$ & $\begin{array}{l}\text { Supra- } \\
\text { tentorial } \\
\text { craniectomy }\end{array}$ & D \\
\hline 10 & 79 & M, $7 \mathrm{~h}$ & Fall,+ & None & $\begin{array}{l}\text { Occip- } \\
\text { ital }\end{array}$ & $7 / 15$ & $\begin{array}{l}15.4 \\
(3.5)\end{array}$ & 2 & $\mathrm{~N}$ & IVH & No lesion & $P$ & $\begin{array}{l}\text { Suboccipital } \\
\text { craniectomy }\end{array}$ & VS \\
\hline 11 & 25 & $\mathrm{M}, 1 \mathrm{~h}$ & MVA,- & None & $\begin{array}{l}\text { Occip- } \\
\text { ital }\end{array}$ & $15 / 15$ & $1(1)$ & 2 & $\mathrm{~N}$ & $N$ & No lesion & A & Conservative & GR \\
\hline 12 & 55 & $\mathrm{M}, 3 \mathrm{~h}$ & MVA,- & $\begin{array}{l}\text { Frac- } \\
\text { ture } \\
\text { clavicle }\end{array}$ & $\begin{array}{l}\text { Occip- } \\
\text { ital }\end{array}$ & $14 / 15$ & \begin{tabular}{|l|}
$5.2(3), 5$ \\
$(2.5)$
\end{tabular} & $\begin{array}{l}2 \text { (bilat- } \\
\text { eral) }\end{array}$ & $\mathrm{N}$ & $\mathrm{N}$ & $\begin{array}{l}\text { Left } \\
\text { temporal } \\
\text { contusion }\end{array}$ & A & Conservative & GR \\
\hline 13 & 55 & $\mathrm{M}, 2 \mathrm{~h}$ & MVA,+ & $\begin{array}{l}\text { Frac- } \\
\text { ture } \\
\text { humer- } \\
\text { us }\end{array}$ & $\begin{array}{l}\text { Occip- } \\
\text { ital }\end{array}$ & $13 / 15$ & $\begin{array}{l}15.4 \\
(3.5)\end{array}$ & 1 & C & IVH & No lesion & A & $\begin{array}{l}\text { Suboccipital } \\
\text { craniectomy }\end{array}$ & MR \\
\hline 14 & 56 & $\mathrm{~F}, 6 \mathrm{~h}$ & MVA,+ & $\begin{array}{l}\text { Frac- } \\
\text { ture } \\
\text { femur }\end{array}$ & $\begin{array}{l}\text { Fron- } \\
\text { tal, } \\
\text { occip- } \\
\text { ital }\end{array}$ & $7 / 15$ & $15(3)$ & 1 & C & C & $\begin{array}{l}\text { Left fron- } \\
\text { totemporal } \\
\text { contusion }\end{array}$ & $P$ & $\begin{array}{l}\text { Suboccipital } \\
\text { and supra- } \\
\text { tentorial } \\
\text { craniectomy }\end{array}$ & D \\
\hline 15 & 25 & $\mathrm{M}, 5 \mathrm{~h}$ & MVA,+ & $\begin{array}{l}\text { Frac- } \\
\text { ture } \\
\text { tibia }\end{array}$ & $\begin{array}{l}\text { Tem- } \\
\text { poral }\end{array}$ & $9 / 15$ & $1(1)$ & 2 & $\mathrm{~N}$ & $N$ & $\begin{array}{l}\text { Right } \\
\text { basifrontal } \\
\text { contusion, } \\
\text { right tem- } \\
\text { poral EDH, } \\
\text { and left } \\
\text { temporal } \\
\text { contusion, } \\
\text { right fron- } \\
\text { to-tempo- } \\
\text { ro-parietal } \\
\text { SDH }\end{array}$ & $P$ & Conservative & SD \\
\hline
\end{tabular}


Table 1 (continued)

\begin{tabular}{|c|c|c|c|c|c|c|c|c|c|c|c|c|c|c|}
\hline $\begin{array}{l}\text { S. } \\
\text { no. }\end{array}$ & $\begin{array}{l}\text { Age } \\
(y)\end{array}$ & $\begin{array}{l}\text { Sex } \\
(M / F) \\
\text { Dura- } \\
\text { tion }^{a}\end{array}$ & $\begin{array}{l}\text { Mode of } \\
\text { injury, } \\
\text { skull } \\
\text { fracture }\end{array}$ & $\begin{array}{l}\text { Asso- } \\
\text { ciated } \\
\text { lesions }\end{array}$ & $\begin{array}{c}\text { Site of } \\
\text { impact }\end{array}$ & $\begin{array}{c}\text { GCS } \\
(3-15)\end{array}$ & $\begin{array}{c}\text { Volume, } \\
\mathrm{cm}^{3} \text {, size } \\
(\mathrm{cm})\end{array}$ & $\begin{array}{c}\text { Type of } \\
\text { location } \\
(1 / 2)\end{array}$ & $\begin{array}{c}\text { Basal } \\
\text { cis- } \\
\text { terns } \\
(\mathrm{N} / \mathrm{C})\end{array}$ & $\begin{array}{c}\text { Fourth } \\
\text { ventricle } \\
(\mathrm{N} / \mathrm{C} / \mathrm{IVH})\end{array}$ & $\begin{array}{c}\text { Associated } \\
\text { supratento- } \\
\text { rial lesions }\end{array}$ & $\begin{array}{l}\text { SAH } \\
(A / P)\end{array}$ & Intervention & GOS \\
\hline 16 & 47 & $\mathrm{M}, 4 \mathrm{~h}$ & Fall,+ & None & Frontal & $3 / 15$ & $2(2)$ & 1 & C & N & $\begin{array}{l}\text { Right } \\
\text { frontal } \\
\text { contusion } \\
\text { with right } \\
\text { frontotem- } \\
\text { poral acute } \\
\text { SDH }\end{array}$ & $\mathrm{P}$ & Conservative & D \\
\hline 17 & 53 & $\mathrm{M}, 6 \mathrm{~h}$ & MVA,- & None & Frontal & $6 / 15$ & $5(2.5)$ & 1 & C & $\mathrm{N}$ & $\begin{array}{l}\text { Bilateral } \\
\text { basifrontal } \\
\text { contusions, } \\
\text { diffuse axo- } \\
\text { nal injury }\end{array}$ & $\mathrm{P}$ & Conservative & $\mathrm{D}$ \\
\hline 18 & 42 & $\mathrm{M}, 6 \mathrm{~h}$ & $\mathrm{MVA},+$ & $\begin{array}{l}\text { None, } \\
\text { chest } \\
\text { injury }\end{array}$ & Frontal & $10 / 15$ & $\begin{array}{l}15.8 \\
(3.5)\end{array}$ & 2 & $\mathrm{~N}$ & $\mathrm{~N}$ & $\begin{array}{l}\text { Right } \\
\text { frontal } \\
\text { contusion } \\
\text { with mild } \\
\text { hydroceph- } \\
\text { alus }\end{array}$ & A & $\begin{array}{l}\text { Suboccipital } \\
\text { craniectomy, } \\
\text { ICD }\end{array}$ & SD \\
\hline 19 & 54 & $\mathrm{M}, 8 \mathrm{~h}$ & Fall,+ & None & $\begin{array}{l}\text { Tem- } \\
\text { poral }\end{array}$ & $5 / 15$ & $12(3)$ & 2 & C & N & $\begin{array}{l}\text { Left tempo- } \\
\text { roparietal } \\
\text { contusion }\end{array}$ & A & Conservative & D \\
\hline 20 & 43 & $\mathrm{M}, 4 \mathrm{~h}$ & MVA,+ & None & $\begin{array}{l}\text { Occip- } \\
\text { ital }\end{array}$ & $9 / 15$ & $4(2)$ & 1 & C & N & $\begin{array}{l}\text { Bilateral } \\
\text { frontal } \\
\text { contusion }\end{array}$ & $P$ & Conservative & SD \\
\hline 21 & 32 & M, $4 \mathrm{~h}$ & MVA,+ & None & $\begin{array}{l}\text { Occip- } \\
\text { ital }\end{array}$ & $15 / 15$ & $5.2(3)$ & 1 & N & $\mathrm{N}$ & $\begin{array}{l}\text { Right } \\
\text { frontal } \\
\text { contusion }\end{array}$ & A & Conservative & GR \\
\hline
\end{tabular}

Abbreviations: A, absent; C, compressed; D, death; DAl, diffuse axonal injury; EDH, extradural hemorrhage; F, female; GCS, Glasgow Coma Scale; GOS, Glasgow Outcome Scale; GR, good recovery; HCP, hydrocephalus; ICD, intercostal tube drainage; IVH, intraventricular hemorrhage; M, male; MD, moderate disability; MVA, motor vehicle accident; N, normal; P, present; SAH, subarachnoid hemorrhage; SD, severe disability; SDH, subdural hemorrhage; VS, vegetative state.

Note: D-Site of impact could not be identified (acceleration-deacceleration injury), skull fracture (+, present;-absent).

aDuration: Timing from injury to reporting to hospital in hours

compared with type 1 . SAH and supratentorial contusions were relatively higher in type 1 hematomas. There was no significant difference in other factors.

\section{Treatment and Outcome}

In our study, 10 patients were treated conservatively and 11 patients underwent surgery. All patients with surgical indications were operated immediately without any delay. Out of 11 patients, 10 patients underwent suboccipital craniectomy and hematoma evacuation, and 1 patient underwent both supratentorial and suboccipital craniectomy. CT head ( - Fig. 3) suggestive of type 2 hematomas were managed conservatively and those suggestive of type 2 hematoma ( - Fig. 1) managed by suboccipital craniectomy with hematoma evacuation. Outcome of 21 patients was GR in 6, MR (moderate recovery) in $3, \mathrm{SD}$ in 3 , VS in 1 , and $\mathrm{D}$ in 8 patients. The incidence of poor outcome was $61.9 \%$ (13/21).

\section{Prognostic Factors}

Frequency, age (range), sex, mode of injury, GCS, hematoma location, basal cisterns, fourth ventricle, SAH, supratentorial contusions, SDH, EDH, IVH, DAI, lesion in posterior fossa, and their relationship with outcome are shown in - Table 3. Agewise distribution shows that there were no females in the favorable group and all females were in the poor outcome group suggesting that females had higher mortality in posterior fossa hematoma.

In our study, GCS at admission was higher in the favorable group as compared with the other group. Brainstem cisterns were compressed in 2 patients in the favorable group as compared with 7 in the poor outcome group. Fourth ventricle was compressed in 1 patient in the favorable group. There was significant presence of SAH in the poor outcome group as compared with the favorable group.

\section{Discussion}

These hematomas approximately constitute one-fourth of all posterior fossa traumatic lesions, ${ }^{3,11}$ but their frequency is almost between SDH and EDH..$^{9,12}$ Sato et $\mathrm{al}^{13}$ established $0.7 \%$ incidence of cerebellar contusions. Nagata et al reported their frequency to be between 0.6 and $0.82 \%$. The incidence observed in our series was $0.41 \%$, which correlates well with other figures. Various mechanisms are responsible for these 
Table 2 Comparison between type 1 and type 2 hematomas

\begin{tabular}{|l|l|l|}
\hline Factors & Type 1 & Type 2 \\
\hline Total patients & $8 / 21$ & $13 / 21$ \\
\hline Age (range), y & $22-56$ & $12-79$ \\
\hline Sex (M/F) & $7 / 1$ & $11 / 2$ \\
\hline Mode of injury (Fall/MVA) & $1 / 7$ & $5 / 8$ \\
\hline Skull fracture (present/absent) & $7 / 1$ & $8 / 5$ \\
\hline Hematoma volume (cm $\left.{ }^{3}\right)$, mean & 6.8 & 14.1 \\
\hline Basal cisterns (N/C) & $1 / 7$ & $11 / 2$ \\
\hline Fourth ventricle (N/C/IVH) & $6 / 1 / 1$ & $9 / 2 / 2$ \\
\hline SAH (P/A) & $6 / 2$ & $4 / 9$ \\
\hline Contusion (P/A) & $7 / 1$ & $4 / 9$ \\
\hline EDH (P/A) & $1 / 7$ & $1 / 12$ \\
\hline SDH (P/A) & $1 / 7$ & $1 / 12$ \\
\hline IVH (P/A) & $0 / 8$ & $1 / 12$ \\
\hline DAI (P/A) & $1 / 7$ & $0 / 13$ \\
\hline SDH in posterior fossa (P/A) & $0 / 8$ & $1 / 12$ \\
\hline
\end{tabular}

Abbreviations: A, absent; C, compressed; DAI, diffuse axonal injury; $\mathrm{EDH}$, extradural hemorrhage; $\mathrm{F}$, female; IVH, intraventricular hemorrhage; M, male; MVA, motor vehicle accident; N, normal; P, present; SAH, subarachnoid hemorrhage, skull fracture (+, present;-, absent); $\mathrm{SDH}$, subdural hemorrhage.

hematomas but remain unclear. These could be coup and countercoup injuries. Coup injuries are thought to be the most frequent mechanism. ${ }^{17,14}$ Countercoup injuries are considered to be rare..$^{15}$

Another mechanism for traumatic intracerebellar hematoma involves acceleration and deacceleration injuries. ${ }^{16}$ In our series, 7/21 patients had coup (33.3), 4/21 (19.0) had countercoup injuries, and the rest (47.6) had accelerationdeacceleration injuries. So, this leads us to the conclusion that acceleration and deacceleration is a common cause as reported previously by Takeuchi et al. ${ }^{17}$

Delayed or evolving hematoma has been reported previously. ${ }^{18,19}$ In our series, no cases of delayed or evolving hematoma was reported as repeat scan was done within 6 hours of admission as mentioned previously. However, repeat CT examination is necessary, as delayed hematomas can develop.

The incidence of poor outcome in previously published series were around 20 to $100 \%$ (average $60 \%$ ), ${ }^{2}$ and in our series, it was $61.9 \%$. The prognosis was better in good GCS patient and in patients who were promptly operated with surgical indication. ${ }^{20}$ Summary of published series of posterior fossa hematomas are depicted in - Table 4 .

D'Avella et al have described protocol for traumatic intracerebellar hematomas as follows:

1. Conservative approach is a treatment option for noncomatose patients with intracerebellar clots $3 \mathrm{~cm}$, except when associated with other EDH or SDH of posterior fossa.

2. Surgery should be done for any patient with any clot $>3 \mathrm{~cm}$.

In our study, surgery was done in 11 patients, out of which 7 patients survived and 4 expired. In our series, type 1
Table 3 Factors related to outcome in traumatic intracerebellar hematoma

\begin{tabular}{|l|l|l|}
\hline Factors & $\begin{array}{l}\text { Favorable } \\
\text { outcome } \\
\text { group }\end{array}$ & $\begin{array}{l}\text { Poor } \\
\text { outcome } \\
\text { group }\end{array}$ \\
\hline Frequency (patients), M/F & $\begin{array}{l}13 / 21, \\
(13 / 0)\end{array}$ & $\begin{array}{l}8 / 21, \\
(5 / 3)\end{array}$ \\
\hline Mode of injury (fall/MVA) & $3 / 10$ & $3 / 5$ \\
\hline Skull fracture (present/absent) & $8 / 5$ & $7 / 1$ \\
\hline GCS (range) & $7-15$ & $3-7$ \\
\hline $\begin{array}{l}\text { Duration (timing from injury } \\
\text { sustained to reporting to hos- } \\
\text { pital), hours, median }\end{array}$ & $4-5 \mathrm{~h}$ & $6 \mathrm{~h}$ \\
\hline Hematoma location (1/2) & $3 / 10$ & $5 / 3$ \\
\hline Hematoma volume (mean) & 12.1 & 10.05 \\
\hline Basal cisterns (N/C) & $11 / 2$ & $1 / 7$ \\
\hline Fourth ventricle (N/C/IVH) & $9 / 1 / 3$ & $6 / 2 / 0$ \\
\hline SAH (P/A) & $3 / 10$ & $7 / 1$ \\
\hline Supratentorial contusion (P/A) & $6 / 7$ & $5 / 3$ \\
\hline HCP (hydrocephalus) & $1 / 12$ & $1 / 7$ \\
\hline Supratentorial EDH (P/A) & $1 / 12$ & $1 / 7$ \\
\hline IVH (P/A) & $0 / 13$ & $1 / 7$ \\
\hline DAI (P/A) & $0 / 13$ & $1 / 7$ \\
\hline SDH in posterior fossa & $1 / 12$ & $0 / 8$ \\
\hline Sbrevions:A, asst; C, & $9 \times 012$ \\
\hline
\end{tabular}

Abbreviations: A, absent; C, compressed; DAl, diffuse axonal injury; EDH, extradural hemorrhage; F, female; GCS, Glasgow Coma Scale; HCP, hydrocephalus; IVH, intraventricular hemorrhage; M, male; MVA, motor vehicle accident; $\mathrm{N}$, normal; $\mathrm{P}$, present; $\mathrm{SAH}$, subarachnoid hemorrhage; SDH, subdural hemorrhage.

aDuration: Timing from injury to reporting to hospital in hours, skull fracture (+, present; - absent).

hematomas were more frequent in the poor outcome group due to easy compression of brainstem or involvement of deep cerebellar nuclei which lead to bad prognosis. ${ }^{17}$

Various factors influencing surgical decision were patients with poor GCS, advanced age, poor general condition, clinical signs of early brainstem damage, bilateral cerebral clots, and associated multiple supratentorial intracranial hemorrhages with evidence of coagulopathy. These factors reflect the peculiar heterogenecity of clinical radiological picture of this subset of posterior fossa hematomas. ${ }^{21}$

HCP is usually not associated with traumatic intracerebellar clots. ${ }^{11,18}$ Karasawa et al ${ }^{3}$ mentioned of acute HCP in $20 \%$ of intracerebellar hematomas, while in our series it was $9.52 \%$. The timing of reaching hospital is important and affects overall morbidity and mortality of patients. Most of the patients in the favorable outcome group reported to hospital within 4 to 5 hours, whereas those in the poor outcome group had reported more than 4 to 8 hours. There was single case of posterior fossa hematoma with associated SDH in our series (-Fig. 1C). This suggests that associated lesions in posterior fossa hematoma such as SDH and EDH are less.

Posterior fossa hematoma is rare in children and incidence of cerebellar hematoma is much rarer. ${ }^{22}$ Most frequent mode 
Table 4 Summary of selected published series of intracerebellar hematoma and contusions

\begin{tabular}{|c|c|c|c|}
\hline Author, year & No. of cases & Notes & Poor outcome (\%) \\
\hline Tsai et al, 1980 & 14 & In 2 cases, associated with brainstem injury & 85 \\
\hline Pozzati et al, 1982 & 7 & All isolated clots & 42 \\
\hline St John et al, 1986 & 3 & One case associated with $\mathrm{AEH}$, one with ASH & 60 \\
\hline $\begin{array}{l}\text { Hamasaki et al, } \\
1987\end{array}$ & 4 & 3 cases isolated with ASH and one with both ASH and AEH & 100 \\
\hline Sato et al, 1987 & 8 & 2 cases with concomitant diffuse cerebral contusions & 50 \\
\hline $\begin{array}{l}\text { Zuccarello et al, } \\
1982\end{array}$ & 5 & All children & 20 \\
\hline Nagata et al, 1991 & 14 & $\begin{array}{l}\text { All delayed hematomas, one personal case, and literature } \\
\text { review }\end{array}$ & 64 \\
\hline Karasawa et al, 1997 & 13 & $\begin{array}{l}11 \text { cases with associated supratentorial ICH, SAH, or ASH; } 2 \\
\text { cases with associated infratentorial ASH }\end{array}$ & 54 \\
\hline D’Avella et al, 2001 & 18 & 8 cases of isolated intracerebellar clots & 50 \\
\hline Present series & 21 & 9 cases of isolated posterior fossa hematoma & 61 \\
\hline
\end{tabular}

Abbreviations: AEH, acute extradural hematoma; ASH, acute subdural hematoma; ICH, intracerebellar hematoma; SAH, subarachnoid hemorrhage.

of injury is fall followed by road traffic accident. ${ }^{23}$ Trauma is a leading cause of childhood head injury, with Center for Disease Control, stating about half a million emergency department visits in the United States is for traumatic brain injury for children aged 0 to 14 years, with 0 to 4 years being the most vulnerable group. ${ }^{24}$ Although most cases are seen in young adults, Zuccarello et $\mathrm{al}^{25}$ reported traumatic posterior fossa hemorrhage in children also. Wright ${ }^{9}$ reported 6 cases and Tsai et al ${ }^{11}$ reported 2 cases of children in their series. Similarly, in our series, we had a single child. Management of such cases is similar to adult posterior fossa hematoma.

Suboccipital craniectomy with hematoma evacuation is the most preferred surgical approach in posterior fossa. ${ }^{26,27}$ Clinical with radiological features, types of hematomas, prognostic factors, and final outcome are discussed and mentioned in -Tables $\mathbf{1 - 3}$. In summary, patients who reported within 4 hours of trauma, with initial GCS score of between 9 and 15, absent SAH, normal basal cisterns, and normal fourth ventricle had better survival rate. However, older age group and females fared worse in our study as shown in - Tables 1-3. In severely ill subjects, surgery should be individualized for each patient with reasonable salvageability.

\section{Conclusion}

Traumatic cerebellar hematoma is a life-threatening condition that requires timely management. Nowadays, with wider availability of CT and intensive neuromonitoring, smaller hematomas can be managed conservatively and larger ones with surgical evacuation in clinically deteriorating patients.

The factors correlated with patient outcome were age, sex, mode of injury, GCS at admission, associated intracranial hematomas, associated SAH, hematoma volume, hematoma location, basal cisterns, status of fourth ventricle, and associated multiple injuries on other body parts. It is hereby concluded that timely surgical intervention should be employed whenever indicated without delay. This study had some limitations, like sample size was relatively small to determine the actual prognostic factors. As these lesions are associated with higher morbidity and mortality, it should be evaluated further to explore pathophysiology for better clinical outcome of patients.

\section{Funding}

None.

\section{Conflict of Interest}

None declared.

\section{References}

1 Patnaik A, Mahapatra AK. Traumatic cerebellar haematoma: a review. The Indian J NeuroTrauma. 2013;10:24-29

2 d'Avella D, Servadei F, Scerrati M, et al. Traumatic intracerebellar hemorrhage: clinicoradiological analysis of 81 patients. Neurosurgery 2002;50(1):16-25, discussion 25-27

3 Karasawa H, Furuya H, Naito H, Sugiyama K, Ueno J, Kin H. Acute hydrocephalus in posterior fossa injury. J Neurosurg 1997;86(4):629-632

4 Liu K. Characteristics of diagnosis and treatment of traumatic intracerebellar hemorrhage [in Chinese] Zhonghua Wai Ke Za Zhi 1997;35(3):166-167

5 Sokol JH, Rowed DW. Traumatic intracerebellar haematoma. Surg Neurol 1978;10(5):340-341

6 Cassinari V, Dorizzi A, Pauli P, Monolo L. Le lesioni traumatiche espansive della fossa cranica posteriore. Su 4 casi di contusione-lacerazione cerebellare. Minerva Neurochir 1967;11(3):230-239

7 Papadakis N, Safran A, Ramirez L, Sujatanond M, Mark VH. Traumatic cerebellar hematoma without subdural hematoma. JAMA 1976;235(5):530-531

8 Schneider RC, Lemmen LJ, Bagchi BK. The syndrome of traumatic intracerebellar hematoma with contrecoup supratentorial complications. J Neurosurg 1953;10(2):122-137

9 Wright RL. Traumatic hematomas of the posterior cranial fossa. J Neurosurg 1966;25(4):402-409

10 Kothari RU, Brott T, Broderick JP, et al. The ABCs of measuring intracerebral hemorrhage volumes. Stroke 1996;27(8):1304-1305 
11 Tsai FY, Teal JS, Itabashi HH, Huprich JE, Hieshima GB, Segall HD. Computed tomography of posterior fossa trauma. J Comput Assist Tomogr 1980;4(3):291-305

12 Bernardi RJ, Smith KR, Jr. Traumatic haematomas. In: Apuzzo MJ, ed. Brain Surgery: Complication Avoidance and Management. Vol. 2. New York, NY: Churchil-Livingstone;1993;1931-1952

13 Sato K, Hinokuma K, Matsuzawa Y, et al. Clinical study of traumatic cerebellar contusion [in Japanese]. No Shinkei Geka 1987;15(12):1285-1289

14 St John JN, French BN. Traumatic hematomas of the posterior fossa. A clinicopathological spectrum. Surg Neurol 1986;25(5):457-466

15 Stone JL, Ladenheim E, Wilkinson SB, Cybulski GR, Oldershaw JB. Hematoma in the posterior fossa secondary to a tangential gunshot wound of the occiput: case report and discussion. Neurosurgery 1991;28(4):603-605, discussion 605-606

16 Kampfl A, Franz G, Aichner F, et al. The persistent vegetative state after closed head injury: clinical and magnetic resonance imaging findings in 42 patients. J Neurosurg 1998;88(5):809-816

17 Takeuchi S, Takasato Y, Masaoka H, Hayakawa T. Traumatic intra-cerebellar haematoma: study of 17 cases. Br. J Neurosurg 2011;25(1):62-67

18 Pozzati E, Grossi C, Padovani R. Traumatic intracerebellar hematomas. J Neurosurg 1982;56(5):691-694

19 Yokota H, Mizunari T, Kuzuhara M, et al. Traumatic delayed intracerebellar hematoma [in Japanese]. Neurol Med Chir (Tokyo) 1988;28(9):886-890

20 Nagata K, Ishikawa T, Ishikawa T, et al. Delayed traumatic intracerebellar hematoma: correlation between the location of the hematoma and the pre-existing cerebellar contusion-case report. Neurol Med Chir (Tokyo) 1991;31(12):792-796

21 D’Avella D, Cacciola F, Angileri FF, et al. Traumatic intracerebellar hemorrhagic contusions and hematomas. J Neurosurg Sci 2001;45(1):29-37

22 Kulshreshtha V, Tripathi P, Jaiswal G, Gupta TK. Traumatic cerebellar haematoma in paedratic patient- a case report and review of literature. Romanian Neurosurg 2016;XXX(4):566-572

23 Adirim TA, Wright JL, Lee E, Lomax TA, Chamberlain JM. Injury surveillance in a pediatric emergency department. Am J Emerg Med 1999;17(6):499-503

24 Centre for Disease Control (CDC). Traumatic brain injury in United States of America: Emergency Department Visits, Hospitalization and Deaths. 2002-2006. Atlanta, GA: CDC2010

25 Zuccarello M, Andrioli GC, Fiore DL, Longatti PL, Pardatscher $\mathrm{K}$, Zampieri P. Traumatic posterior fossa haemorrhage in children. Acta Neurochir (Wien) 1982;62(1-2):79-85

26 Pollak L, Rabey JM, Gur R, Schiffer J. Indication to surgical management of cerebellar hemorrhage. Clin Neurol Neurosurg 1998;100(2):99-103

27 Aghi M, Ogilvy CS, Carter B, Surgical management of intacerebellar haemorrhage. In Roberts DW, Schmeidek HH, eds. Schmeidek and Sweet's Operative Neurosurgical Techniques, Indications, Methods, Results. Vol. 2, 5th ed. Philadelphia: Saunders/Elsevier2005;1061-1074

28 Hamasaki T, Yamaki T, Yoshino E, et al. Traumatic posterior fossa hematoma. No To Shinkei. 1987;39(11):1083-1090 